\title{
Increased $\gamma$-glutamyl transferase positively contributed to the poor prognosis in lung cancer patients
}

\author{
Jing Jing Ran ${ }^{1}$, Ruixin $\mathrm{He}^{2}$, Qiang $\mathrm{Yu}^{2}$, Limei Kang ${ }^{2}$, Yaoqi Fu ${ }^{2}$, Cong Bo ${ }^{2}$, Li Zhang ${ }^{1}$ \\ ${ }^{1}$ Laboratory of Pathology, Key Laboratory of Transplantation Engineering and Immunology, Ministry of Health, West China Hospital, Sichuan \\ University, Chengdu 610041, China; ${ }^{2}$ West China Medical School, Sichuan University, Chengdu 610041, China \\ Contributions: (I) Conception and design: L Zhang, JJ Ran, R He; (II) Administrative support: None; (III) Provision of study materials or patients: L \\ Zhang, JJ Ran; (IV) Collection and assembly of data: JJ Ran, Q Yu, L Kang, Y Fu, C Bo; (V) Data analysis and interpretation: L Zhang, JJ Ran; (VI) \\ Manuscript writing: All authors; (VII) Final approval of manuscript: All authors. \\ Correspondence to: Li Zhang. Laboratory of Pathology, Key Laboratory of Transplantation Engineering and Immunology, Ministry of Health, West \\ China Hospital, Sichuan University, Chengdu 610041, China. Email: zhangli7375@scu.edu.cn.
}

\begin{abstract}
Background: $\gamma$-glutamyl transferase (GGT) has a critical effect on tumor initiation, progression, and metastasis; however, the particular role of circulating GGT factor in the prognosis of lung cancer remains undetermined. The aim of this study is to identify the relationship between the circulating level of GGT and poor prognosis of lung cancer patients.

Methods: A total of 1,098 lung cancer patients whose GGT values were available were enrolled in this study and divided into positive and negative groups. SPSS 19.0 was introduced to analyze the relationship between GGT and clinical characteristics and metastasis. The relationship between survival status and GGT was completed by Kaplan-Meier method. Cox regression model was conducted to confirm whether GGT served as an independent risk factor in the prognosis of lung cancer.

Results: Elevated GGT level was positively related to liver $(\mathrm{P}<0.01)$, bone $(\mathrm{P}<0.01)$, and lymph node $(\mathrm{P}<0.05)$ metastasis. Kaplan-Meier analysis indicated that elevated GGT significantly contributed to poor survival of lung cancer. In the specific histological subtype analysis, we found that GGT was only positively related to the survival condition of small-cell lung cancer $(\mathrm{P}<0.01)$, but not adenocarcinoma (ADC) $(\mathrm{P}=0.08)$ or squamous carcinoma (SCC) $(\mathrm{P}=0.49)$. Multivariate Cox regression model indicated that GGT could act as an independent factor for prediction of poor prognosis in lung cancer.
\end{abstract}

Conclusions: Our results confirmed that GGT contributed to poor survival of lung cancer and was important in prediction of metastasis and poor prognosis.

Keywords: $\gamma$-glutamyl transferase (GGT); lung cancer; survival status; poor prognosis

Submitted Jul 28, 2018. Accepted for publication Nov 02, 2018.

doi: $10.21037 /$ tcr.2018.11.11

View this article at: http://dx.doi.org/10.21037/tcr.2018.11.11

\section{Introduction}

Lung cancer has the highest mortality rates among all cancers due to its late diagnosis (1). The routine techniques for lung cancer diagnosis include low-dose computed tomography (LDCT), sputum cytology testing and chest X-ray (2). While a LDCT can detect tumors at an earlier stage and help reduce the tumor mortality rate by up to $20 \%$ (3), CT-based diagnosis of lung cancer has a significant false-positive rate (4). Meanwhile, traditional serum biomarkers, such as neuron-specific enolase (NSE) (5), carbohydrate antigen 125 (6) and carcinoembryonic antigen (CEA), play critical roles in the diagnosis and prognosis of lung cancer patients despite their low sensitivity and specificity which range between $50 \%$ and $60 \%(7,8)$. Thus, novel, economical and noninvasive biomarkers are urgently needed for early diagnosis of lung cancer (9).

The enzyme, $\gamma$-glutamyl transferase (GGT), is associated with many disease outcomes (10) and has a prognostic 
value for various cancers (11). Ma et al. reported that elevated serum GGT levels in hepatocellular carcinoma patients treated with radiofrequency ablation may serve as a prognostic marker to predict significantly decreased overall survival (OS) and recurrence (12). A study of several serum biomarkers in metastatic colorectal cancer, including GGT, alkaline phosphatase, and CEA, showed that combination of GGT and CEA have prognostic value to predict overall and progression-free survival (13). Kunutsor and Laukkanen found that increased GGT level contributed to poor prognosis in middle-aged prostate cancer patients (14), while another study also indicated that serum GGT level is a novel independent factor for poor prognosis of renal cell carcinoma (15).

However, a systematic analysis of the association between circulating GGT and the survival status of lung cancer patients has not yet been conducted. The present study retrospectively investigated GGT serum levels in Chinese lung cancer patients to determine whether it can be feasibly used as a prognostic parameter.

\section{Methods}

\section{Patients}

Lung cancer patients treated at the Pneumology Department of West China Hospital (Chengdu, China) between January 2008 and July 2012 were enrolled in the present study. All patient medical information was obtained from a prospective database. Exclusion criteria included lack of GGT level data, pathological cancer confirmation, follow-up data, evidence of lung cancer metastasis. Metastasis was determined by biopsy and whole-body CT scan. Survival time was determined as the time from disease diagnosis to the date of death or last follow-up by telephone inquiry. Tumor stages were defined according to the $7^{\text {th }}$ edition of Tumor-Node-Metastasis staging (16). Subtype classification of lung cancer, especially ADC, was based on the 2015 WHO classification (17).

\section{Clinical design}

Serum GGT concentration was assessed by an immunoassay in the Medical Laboratory Department of the West China Hospital before surgery or any other treatment. Patients were classified into two groups (positive and negative) based on a serum GGT cut-off value of $40 \mathrm{U} / \mathrm{L}$, which is widely recognized as the normal level. Patients in the positive group had a serum GGT level of $\geq 40 \mathrm{U} / \mathrm{L}$, whereas patients in the negative group had a GGT level of $<40 \mathrm{U} / \mathrm{L}$. A correlation analysis of pretreatment serum GGT levels and clinical parameters (e.g., gender, age, smoking status, tumor type, tumor stage, and distant metastasis) was performed for patients classified as having lung cancer, adenocarcinoma (ADC), and squamous cell carcinoma (SCC). The relationship between serum GGT levels and survival prognosis was also investigated. A multivariate Cox regression model was used to determine whether serum GGT levels could serve as a prognostic parameter for lung cancer.

\section{GGT measurement}

Before operation initiation, as part the of routine pretreatment, blood samples were drawn so as to evaluate the hepatic damage, and other biochemical tests. The period occurred about 24 to 48 hours before starting the specific therapy by way of a peripheral venous puncture. We examined and analyzed GGT concentrations using an enzymatic colorimetric test at $37^{\circ} \mathrm{C}$. The identical conditions were used for L-g-glutamyl-3-carboxy-4nitroanilide substrate (18).

\section{Statistical analysis}

SPSS version 19.0 was used to analyze all obtained data. A Chi-square test was used to evaluate the intergroup differences for discontinuous data. Survival status was analyzed by Kaplan-Meier curve and a multivariate Cox regression hazard ratio (HR) model was used to identify independent prognostic factors by analyzing clinical characteristics, and metastasis survival status. $\mathrm{P}<0.05$ was considered statistically significant.

\section{Results}

\section{Relationship between serum GGT levels and metastasis}

A total of 1,098 patients with lung cancer were included in the present study and classified into the GGTpositive ( $\mathrm{N}=320)$ and -negative $(\mathrm{N}=778)$ groups, based on a pretreatment serum GGT level cutoff of $40 \mathrm{U} / \mathrm{L}$. Demographic and clinicopathological parameters of all enrolled patients are shown in Table 1. GGT stratification analysis showed very significant differences between the two groups with respect to clinicopathological features, such as gender $(\mathrm{P}<0.001)$, smoking status $(\mathrm{P}<0.001)$, and tumor 
Table 1 Demographics and clinical pathological features of all enrolled lung cancer patients

\begin{tabular}{lc}
\hline Characteristics & No. of patients $(\%)(\mathrm{n}=1,098)$ \\
\hline Gender & $754(68.7)$ \\
Male & $344(31.3)$ \\
Female & \\
Age & $93(8.5)$ \\
$<45$ & $643(58.6)$ \\
$45-65$ & $362(33.0)$ \\
$>65$ & \\
Histological classification & $290(26.4)$ \\
Adenocarcinoma (ADC) & $581(52.9)$ \\
Squamous (SCC) & $195(17.8)$ \\
SCLC & $32(2.9)$ \\
Others & \\
Stage & \\
I & $103(9.4)$ \\
II & $106(9.7)$ \\
III & $283(25.8)$ \\
IV & $606(55.2)$ \\
Netastasis & \\
No & \\
GGT & \\
Negative & $(29.1)$ \\
\hline
\end{tabular}

SCLC, small cell lung cancer; GGT, $\gamma$-glutamyl transferase.

stage $(\mathrm{P}<0.01)$, but not histological classification $(\mathrm{P}=0.164)$ or age $(\mathrm{P}=0.211)$. Serum GGT levels were also closely related to metastasis to bone (positive: $37.9 \%$, negative: $62.1 \%$; $\mathrm{P}<0.01$ ), liver (positive: $42.2 \%$, negative: $57.8 \%$; $\mathrm{P}<0.01$ ), and lymph nodes (positive: $31.7 \%$, negative: $68.3 \% ; \mathrm{P}<0.05$ ) (Table 2).

\section{Correlation between GGT levels and metastasis incidence in $A D C$ and SCC}

Lung cancer primarily consists of two subtypes: nonsmall cell lung cancer (NSCLC) and small cell lung cancer (SCLC). NSCLC cases were mainly composed of
$\mathrm{ADC}$ and squamous carcinoma (SCC). We next analyzed the association between the serum GGT levels, clinical characteristics, and metastasis in ADC patients. The results showed a pronounced correlation with the presence of metastatic lesions in bone (negative: $56.3 \%$, positive: 43.8 , $\mathrm{P}<0.01$ ), liver (negative: $54.2 \%$, positive: $45.8, \mathrm{P}<0.05$ ), and lymph nodes (negative: $63.3 \%$, positive: $36.7, \mathrm{P}<0.01$ ). However, GGT-positive levels were not related to clinical features, such as gender $(\mathrm{P}=0.952)$, smoking status $(\mathrm{P}=0.902)$, and tumor stage $(\mathrm{P}=0.183)$ (Table 3). Overall, a total of 290 patients (negative: 195, positive: 95 ) were diagnosed with SCC. Histological subtype analysis showed that elevated serum GGT levels were not significantly correlated with clinicopathological parameters or diverse metastasis occurrence (all $\mathrm{P}>0.05$ ) (Table 4) in SCC patients.

\section{Association between serum GGT levels, lung cancer, clinical characteristics, and survival}

Kaplan-Meier survival curves played a more important role in the assessment of the mortality at 3-5 years. Fiveyear survival analysis, specifically follow-ups ranging from 1 to 60 months, confirmed that high concentrations of serum GGT were closely associated with survival of all lung cancer patients $(\mathrm{P}<0.01)$. In particular, elevated serum GGT levels were significantly associated with survival of SCLC patients $(\mathrm{P}<0.01)$, but not ADC $(\mathrm{P}=0.08)$ or SCC $(\mathrm{P}=0.49)$ (Figure 1A). With respect to clinical characteristics, serum GGT levels were significantly correlated with survival in males $(\mathrm{P}<0.01)$, non-smokers $(\mathrm{P}<0.01)$, and tumor stages III-IV $(\mathrm{P}<0.01)$. Serum GGT levels were not significantly associated with any other clinical features (Figure 1B,C).

\section{Independent factors for predicting a poor prognosis}

By multivariate Cox regression model, based on the cut-off value for GGT level, we attempted to determine whether serum GGT levels can serve as an independent factor for predicting a poor prognosis in lung cancer patients. A multivariate Cox regression model can be used to analyze associations between multiple factors and OS times of patients; and, as is shown in Table 5, a significant difference was found for metastasis [95\% confidence interval (CI): $1.196-1.814 ; \mathrm{P}<0.001]$. Table 5 provides additional information concerning the multi-variable survival analysis. Compared with the GGT-negative group, the HR of GGT-positive patients increased by 0.858 , with a $95 \%$ CI (0.730-1.009) $(\mathrm{P}=0.064)$. The HR value of all lung cancer 
Table 2 Correlation of clinical characteristics and metastasis after stratification analysis by serum GGT levels

\begin{tabular}{|c|c|c|c|c|}
\hline Variables & Negative (\%) (n=778) & Positive (\%) $(n=320)$ & Total $(n=1,098)$ & $P$ value \\
\hline \multicolumn{5}{|l|}{ Basic characteristics } \\
\hline \multicolumn{5}{|l|}{ Age } \\
\hline$<45$ years & $60(64.5)$ & $33(35.5)$ & 93 & 0.211 \\
\hline $45-65$ years & $452(70.3)$ & $191(29.7)$ & 643 & \\
\hline \multicolumn{5}{|l|}{ Gender } \\
\hline Male & 509 (67.5) & $245(32.5)$ & 754 & $<0.001^{\star \star \star}$ \\
\hline Female & $269(78.2)$ & $75(21.8)$ & 344 & \\
\hline \multicolumn{5}{|l|}{ Histological classification } \\
\hline SCLC & $132(67.7)$ & $63(32.3)$ & 195 & \\
\hline Others & $22(68.8)$ & $10(31.3)$ & 32 & \\
\hline \multicolumn{5}{|l|}{ Smoke status } \\
\hline No & $364(76.3)$ & $113(23.7)$ & 477 & $<0.001^{\star \star \star}$ \\
\hline Yes & $414(66.7)$ & 207 (33.3) & 621 & \\
\hline \multicolumn{5}{|l|}{ Stage } \\
\hline 1 & $76(73.8)$ & $27(26.2)$ & 103 & $<0.01^{\star \star}$ \\
\hline II & 83 (78.3) & $23(21.7)$ & 106 & \\
\hline \multicolumn{5}{|l|}{ Metastasis } \\
\hline No & $694(71.4)$ & $278(28.6)$ & 972 & 0.271 \\
\hline Yes & $84(66.7)$ & $42(33.3)$ & 126 & \\
\hline \multicolumn{5}{|l|}{ Bone } \\
\hline No & $639(73.1)$ & $235(26.9)$ & 874 & $<0.01^{\star *}$ \\
\hline Yes & $139(62.1)$ & 85 (37.9) & 224 & \\
\hline \multicolumn{5}{|l|}{ Liver } \\
\hline No & 719 (72.2) & 277 (27.8) & 996 & $<0.01^{\star \star}$ \\
\hline Yes & $59(57.8)$ & $43(42.2)$ & 102 & \\
\hline \multicolumn{5}{|l|}{ Adrenal gland } \\
\hline No & $739(71.3)$ & $298(28.7)$ & 1,037 & 0.221 \\
\hline Yes & 39 (63.9) & $22(36.1)$ & 61 & \\
\hline
\end{tabular}

Table 2 (continued) 
Table 2 (continued)

\begin{tabular}{|c|c|c|c|c|}
\hline Variables & Negative (\%) $(\mathrm{n}=778)$ & Positive (\%) (n=320) & Total $(n=1,098)$ & $P$ value \\
\hline No & $334(74.6)$ & $114(25.4)$ & 448 & $<0.05^{\star}$ \\
\hline Yes & $444(68.3)$ & $206(31.7)$ & 650 & \\
\hline \multicolumn{5}{|c|}{ Intrapulmonary } \\
\hline Yes & $89(67.4)$ & $43(32.6)$ & 132 & \\
\hline \multicolumn{5}{|l|}{ Pleural } \\
\hline No & $662(71.0)$ & $271(29.0)$ & 933 & 0.865 \\
\hline Yes & $116(70.3)$ & $49(29.7)$ & 165 & \\
\hline Yes & $20(64.5)$ & $11(35.5)$ & 31 & \\
\hline
\end{tabular}

$\mathrm{P}$ values were calculated using the Chi-square test. ${ }^{*}, \mathrm{P}<0.05 ;{ }^{* *}, \mathrm{P}<0.01 ;{ }^{* \star *}, \mathrm{P}<0.001$. GGT, $\gamma$-glutamyl transferase.

patients older than 65 years increased to 0.659 (95\% CI: 0.562-0.773, $\mathrm{P}<0.001)$. Meanwhile, smoking history (HR, $1.202,95 \%$ CI: $1.029-1.404, \mathrm{P}<0.05)$ and advanced tumor stage (III: HR, 0.492, 95\% CI: 0.360-0.671; IV: HR, 0.736, 95\% CI: 0.616-0.880) were identified as independent risk factors for predicting poor outcomes (Table 5).

Specific histological subtype analysis had revealed similar results for the ADC patients. Other factors that could be used to predict a poor outcome in ADC patients included being $\geq 65$ years old (HR, 0.704, 95\% CI: 0.560-0.886), a smoking history (HR, $1.271,95 \%$ CI: $1.028-1.571$ ), tumor stages II-IV (II: HR, 0.180; III: HR, 0.437; IV: HR, 0.735), and presence of metastases (HR, 1.583, 95\% CI: $1.153-2.173)$. The HR also increased by 0.927 in the GGT-positive group (95\% CI: 0.734-1.170, P=0.521). Furthermore, the results demonstrated that serum GGT levels could be employed as a crucial biomarker in ADC patients (Table 6). Nevertheless, only elevated GGT levels identified a poor prognosis factor for SCLC.

\section{Discussion}

Although the prognostic value of serum GGT level in several tumors has been identified, the value concerning poor prognosis for lung cancer has not yet been determined. In our study, a large-scale population was classified into negative and positive groups. Here, we found that elevated serum GGT levels were more strongly associated with clinicopathological parameters, such as gender $(\mathrm{P}<0.001)$, smoking status $(\mathrm{P}<0.001)$, and tumor stage $(\mathrm{P}<0.01)$. Other clinical-pathological features showed no significant difference. Moreover, serum GGT levels showed a dramatic correlation with metastasis to the liver $(\mathrm{P}<0.01)$, bone $(\mathrm{P}<0.01)$, and lymph nodes $(\mathrm{P}<0.05)$ (Table 2$)$, and a similar relationship was even more pronounced for ADC patients. The results revealed that high serum GGT levels were positively associated with metastasis (bone: $\mathrm{P}<0.01$; liver: $\mathrm{P}<0.05$; lymph node: $\mathrm{P}<0.01$ ) (Table 3). Elevated GGT levels also positively correlated with the patient's OS rate for all lung cancer and subtypes $(\mathrm{P}<0.01)$, specifically SCLC $(\mathrm{P}<0.01)$ but not with $\mathrm{ADC}$ or $\mathrm{SCC}$, males $(\mathrm{P}<0.01)$, smoking status $(\mathrm{P}<0.01)$, and tumor stage $(\mathrm{P}<0.01)$ (Figure $1 A, B, C)$. These results are mostly consistent with previous studies in sex difference. In one study of serum GGT and coronary artery calcification (CAC) (19), elevated GGT level was an independent and reliable indicator for males but not females, with the key difference being attributed to more alcohol consumption in men compared with women (20). However, there is another interesting study with contrasting results. In Kim's study, the results revealed that elevated GGT level indicated poor clinical outcomes in females (21). In addition, GGT was strongly associated with incidence of tumor. It has also been reported that GTT can mediate GSH metabolism to produce lipid peroxidation, thus 
Table 3 Association of clinical characteristics and metastasis stratified by GGT levels in adenocarcinoma patients (ADC)

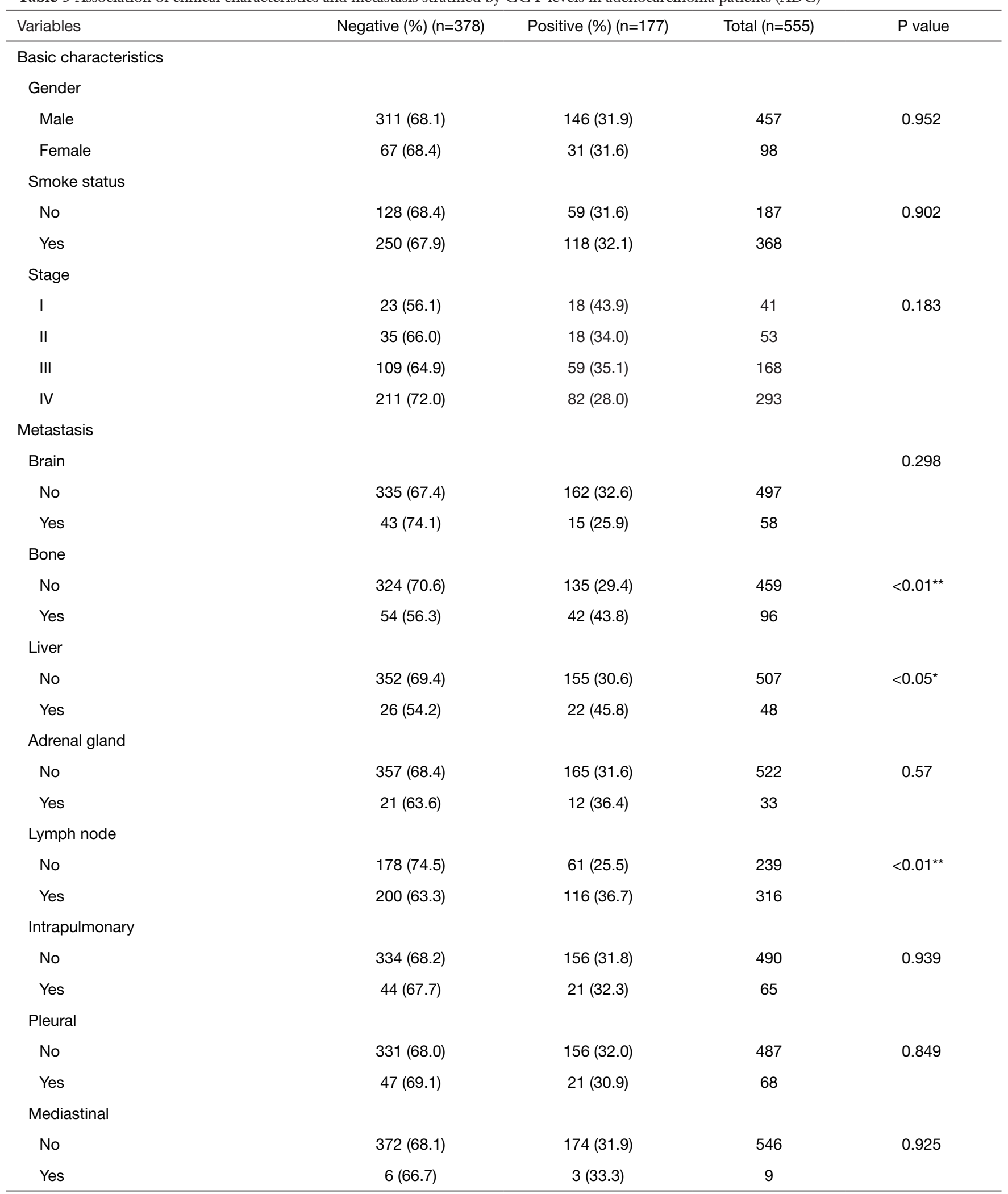

$\mathrm{P}$ values were calculated using the Chi-square test. ${ }^{*}, \mathrm{P}<0.05 ;{ }^{* *}, \mathrm{P}<0.01 . \mathrm{GGT}, \gamma$-glutamyl transferase. 
Table 4 Association of clinical characteristics and metastasis stratified by GGT levels in squamous carcinoma patients (SCC)

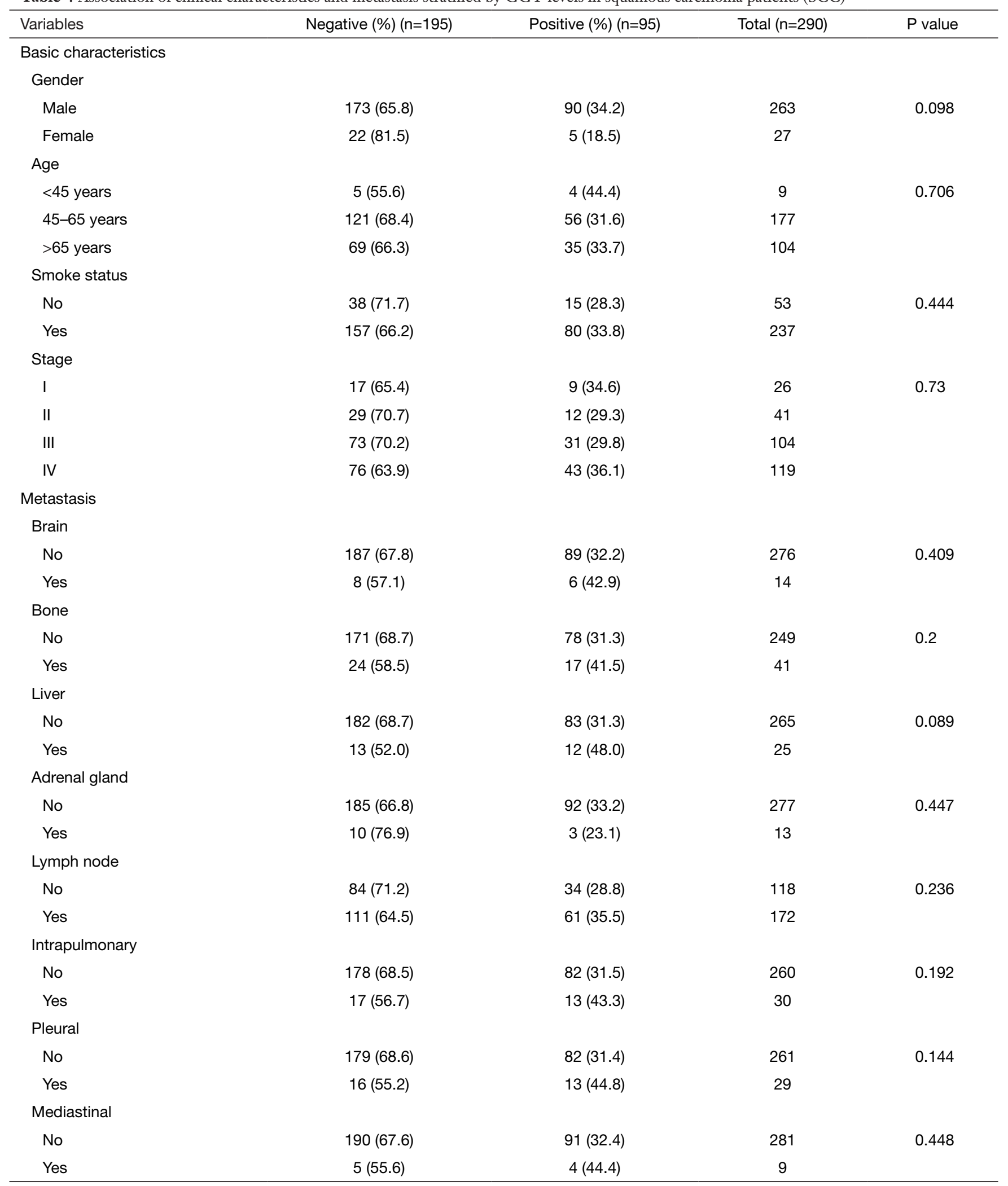

GGT, $\gamma$-glutamyl transferase. 

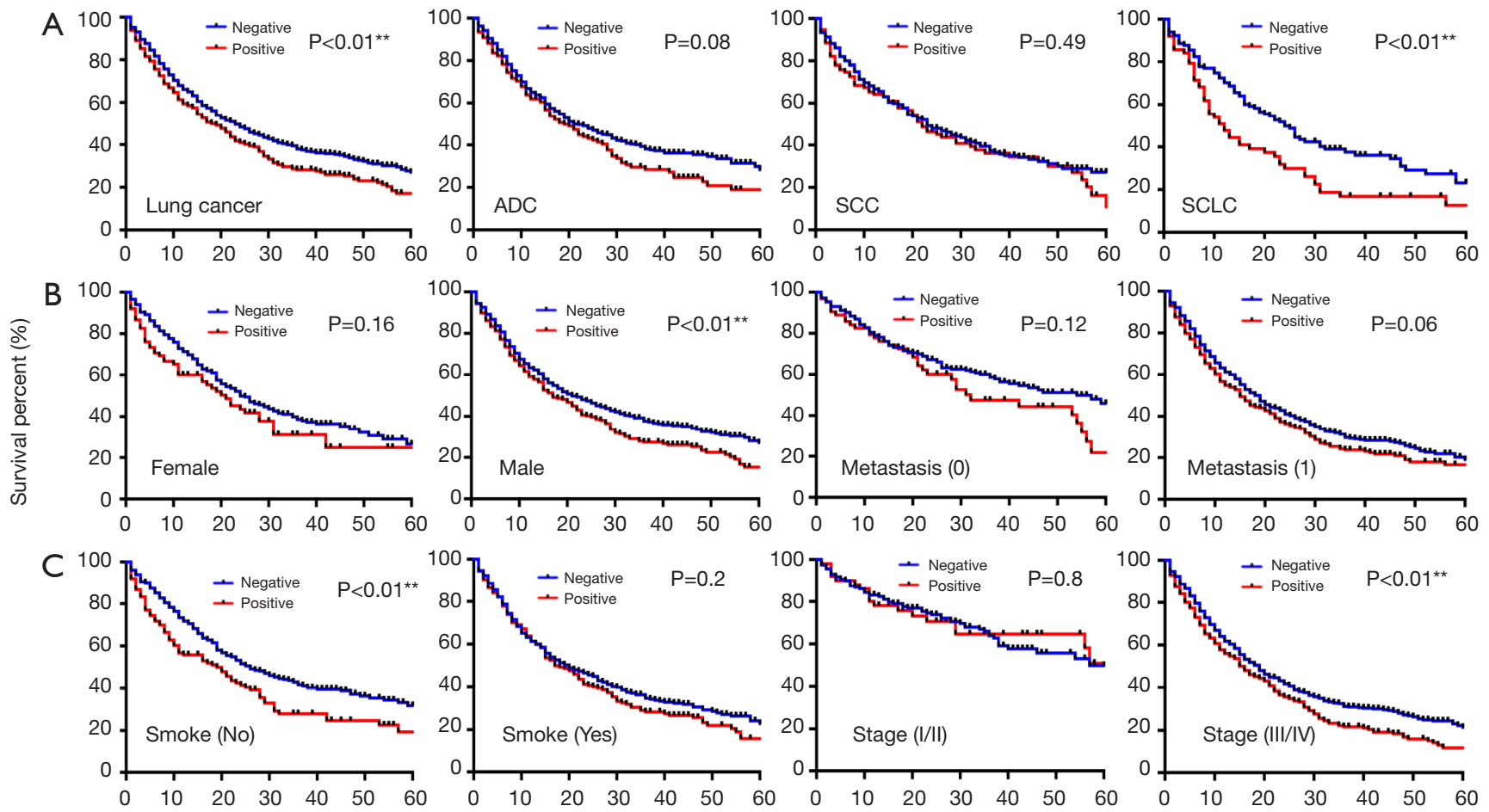

Figure 1 The survival status of lung cancer patients and clinicopathological parameters correlated with GGT. (A) All lung cancer patients, ADC patients, SCC patients, SCLC patients; (B) female/male/metastasis (0/1) patients; (C) smoke (no/yes), stage (I/II) and stage (III/IV). GGT, $\gamma$-glutamyl transferase; ADC, adenocarcinoma; SCC, squamous carcinoma; SCLC, small cell lung cancer.

Table 5 Multivariate analysis for all lung cancer

\begin{tabular}{|c|c|c|c|c|}
\hline Variables & SE & $\operatorname{Exp}(B)$ & $95 \% \mathrm{Cl}$ for $\operatorname{Exp}(\mathrm{B})$ & $P$ value \\
\hline \multicolumn{5}{|l|}{$<45$} \\
\hline $45-65$ & 0.149 & 0.645 & $0.482-0.863$ & $<0.01^{\star \star}$ \\
\hline$>65$ & 0.081 & 0.659 & $0.562-0.773$ & $<0.001^{\star * *}$ \\
\hline \multicolumn{5}{|l|}{ Stages } \\
\hline \multicolumn{5}{|l|}{ I } \\
\hline II & 0.199 & 0.326 & $0.220-0.481$ & \\
\hline III & 0.159 & 0.492 & $0.360-0.671$ & $<0.001^{\star \star \star}$ \\
\hline GGT (negative/positive) & 0.083 & 0.858 & $0.730-1.009$ & 0.064 \\
\hline
\end{tabular}

$\mathrm{P}$ values were calculated using the Chi-square test. ${ }^{* \star}, \mathrm{P}<0.01 ;{ }^{* \star *}, \mathrm{P}<0.001$. GGT, $\gamma$-glutamyl transferase. 
Table 6 Multivariate analysis for adenocarcinoma patients

\begin{tabular}{|c|c|c|c|c|}
\hline Variables & SE & $\operatorname{Exp}(B)$ & $95 \% \mathrm{Cl}$ for $\operatorname{Exp}(\mathrm{B})$ & $P$ value \\
\hline \multicolumn{5}{|l|}{$<45$} \\
\hline $45-65$ & 0.182 & 0.652 & $0.456-0.931$ & $<0.05^{\star}$ \\
\hline$>65$ & 0.117 & 0.704 & $0.560-0.886$ & 0.01 \\
\hline \multicolumn{5}{|l|}{ Stages } \\
\hline \multicolumn{5}{|l|}{ I } \\
\hline II & 0.338 & 0.18 & $0.093-0.350$ & $<0.001^{\star \star \star}$ \\
\hline III & 0.256 & 0.437 & $0.265-0.721$ & $<0.01^{\star \star}$ \\
\hline GGT (negative/positive) & 0.119 & 0.927 & $0.734-1.170$ & 0.521 \\
\hline
\end{tabular}

$\mathrm{P}$ values were calculated using the Chi-square test. ${ }^{*}, \mathrm{P}<0.05 ;{ }^{* *}, \mathrm{P}<0.01 ;{ }^{* \star *}, \mathrm{P}<0.001$. GGT, $\gamma$-glutamyl transferase.

explaining the correlation between high GGT and poor prognosis (22). Furthermore, serum GGT levels were found that is not independent poorly prognostic factor (Table 5).

GGT is a well-known marker of apoptosis and cellular detoxification (23) which has a remarkable relationship with metabolic syndrome, cardiovascular disease and diabetes (24). Highly active enzymes, like GGT, are secreted from organs, such as the liver, kidneys, and pancreas (25). An increasing number of clinical studies have demonstrated there to be an association between GGT and a poor survival (26) rate. Previously, Staudigl et al. showed that serum GGT levels could serve as a distinct prognostic parameter for breast cancer patients with metastases (18). In an analysis of 411 intrahepatic cholangiocarcinoma patients, Yin et al. found that high serum GGT levels could serve as a poor prognostic indicator for predicting invasive tumor behaviors (27). Yang et al. showed that serum GGT levels correlated with poor prognosis and the development of high-grade esophageal epithelial dysplasia in 639 patients (28). In patients with non-metastatic renal cell carcinoma with venous tumor thrombus, Luo et al. demonstrated that pre-operative GGT levels could act as an independent prognostic indicator (29). Elevated GGT levels have been observed to be crucial risk factor in a study of endometrial cancer survival biomarker which related with lifestyle (30). Mechanistically, in terms of tumorigenesis, the exact mechanism for correlating the elevated GGT levels and a vascular invasion with a lymph node involvement (31) remains unknown. It has been suggested that metastatic activity might be promoted by overexpression of GGT in melanoma, which regulates extracellular and intracellular GSH metabolism (32). It is possible that elevated GGT levels promote tumor growth by this method. While a large number of reports have confirmed the link between elevated serum GGT levels and tumor initiation, progression, recurrence, and metastasis, there is still an urgent need to further clarify the complete role of GGT in carcinogenesis (33). There were several potential limitations in this study. A portion of the patients were excluded due to incomplete data acquisition and the retrospective study design, and others were excluded due to alcohol abuse and hepatobiliary-diseases, which affected their serum GGT levels. Nevertheless, our results have significant clinical relevance.

To our knowledge, this study is the first to report the prognostic value of elevated circulating GGT levels in a large-scale population of lung cancer patients. Our findings confirmed that serum GGT levels play a crucial role in lung cancer susceptibility and can serve as a valuable prognostic indicator, especially in SCLC patients.

\section{Conclusions}

In conclusion, we designed this study to ascertain whether or not the GGT levels positively correlate with the occurrence of metastasis and risk survival. Our results demonstrated that GGT contributed to poor survival of 
lung cancer and was crucially informative in the prediction of metastasis and poor prognosis. Further research is needed to determine if an understanding of the mechanistic pathways of GGT might have potential value in lung cancer prevention.

\section{Acknowledgments}

Funding: This work was supported by the National Natural Science Foundation of China (document No. 81772478), and the Science and Technology Project of Chengdu (2017-CY02-00031-GX).

\section{Footnote}

Conflicts of Interest: All authors have completed the ICMJE uniform disclosure form (available at http://dx.doi. org/10.21037/tcr.2018.11.11). The authors have no conflicts of interest to declare.

Ethical Statement: The authors are accountable for all aspects of the work in ensuring that questions related to the accuracy or integrity of any part of the work are appropriately investigated and resolved. The study was conducted in accordance with the Declaration of Helsinki (as revised in 2013). This study was approved by the Biomedical Ethics Committee Subcommittee in West China Hospital of Sichuan University. Informed consent was waived.

Open Access Statement: This is an Open Access article distributed in accordance with the Creative Commons Attribution-NonCommercial-NoDerivs 4.0 International License (CC BY-NC-ND 4.0), which permits the noncommercial replication and distribution of the article with the strict proviso that no changes or edits are made and the original work is properly cited (including links to both the formal publication through the relevant DOI and the license). See: https://creativecommons.org/licenses/by-nc-nd/4.0/.

\section{References}

1. Broodman I, Lindemans J, van Sten J, et al. Serum Protein Markers for the Early Detection of Lung Cancer: A Focus on Autoantibodies. J Proteome Res 2017;16:3-13.

2. Oudkerk M, Devaraj A, Vliegenthart R, et al. European position statement on lung cancer screening. Lancet Oncol 2017;18:e754-66.

3. Blanchon T, Brechot JM, Grenier PA, et al. Baseline results of the Depiscan study: a French randomized pilot trial of lung cancer screening comparing low dose CT scan (LDCT) and chest X-ray (CXR). Lung Cancer 2007;58:50-8.

4. van den Bergh KA, Essink-Bot ML, Bunge EM, et al. Impact of computed tomography screening for lung cancer on participants in a randomized controlled trial (NELSON trial). Cancer 2008;113:396-404.

5. Feng Q, Yu M, Kiviat NB. Molecular biomarkers for cancer detection in blood and bodily fluids. Crit Rev Clin Lab Sci 2006;43:497-560.

6. Ghosh I, Bhattacharjee D, Das AK, et al. Diagnostic Role of Tumour Markers CEA, CA15-3, CA19-9 and CA125 in Lung Cancer. Indian J Clin Biochem 2013;28:24-9.

7. Gao WM, Kuick R, Orchekowski RP, et al. Distinctive serum protein profiles involving abundant proteins in lung cancer patients based upon antibody microarray analysis. BMC Cancer 2005;5:110.

8. Patz EF, Jr., Campa MJ, Gottlin EB, et al. Biomarkers to help guide management of patients with pulmonary nodules. Am J Respir Crit Care Med 2013;188:461-5.

9. Vendrell JA, Mau-Them FT, Beganton B, et al. Circulating Cell Free Tumor DNA Detection as a Routine Tool forLung Cancer Patient Management. Int J Mol Sci 2017;18. doi: 10.3390/ijms18020264.

10. Griffith OW, Bridges RJ, Meister A. Transport of gammaglutamyl amino acids: role of glutathione and gammaglutamyl transpeptidase. Proc Natl Acad Sci U S A 1979;76:6319-22.

11. Wu SJ, Lin YX, Ye H, et al. Prognostic value of alkaline phosphatase, gamma-glutamyl transpeptidase and lactate dehydrogenase in hepatocellular carcinoma patients treated with liver resection. Int J Surg 2016;36:143-51.

12. Ma H, Zhang L, Tang B, et al. gammaGlutamyltranspeptidase is a prognostic marker of survival and recurrence in radiofrequency-ablation treatment of hepatocellular carcinoma. Ann Surg Oncol 2014;21:3084-9.

13. He WZ, Guo GF, Yin CX, et al. Gamma-glutamyl transpeptidase level is a novel adverse prognostic indicator in human metastatic colorectal cancer. Colorectal Dis 2013;15:e443-52.

14. Kunutsor SK, Laukkanen JA. Gamma-glutamyltransferase and risk of prostate cancer: Findings from the KIHD prospective cohort study. Int J Cancer 2017;140:818-24.

15. Hofbauer SL, Stangl KI, de Martino M, et al. Pretherapeutic gamma-glutamyltransferase is an independent prognostic factor for patients with renal cell carcinoma. Br J Cancer 2014;111:1526-31. 
16. Goldstraw P, Chansky K, Crowley J, et al. The IASLC Lung Cancer Staging Project: Proposals for Revision of the TNM Stage Groupings in the Forthcoming (Eighth) Edition of the TNM Classification for Lung Cancer. J Thorac Oncol 2016;11:39-51.

17. Travis WD. The 2015 WHO classification of lung tumors. Pathologe 2014;35 Suppl 2:188.

18. Staudigl C, Concin N, Grimm C, et al. Prognostic relevance of pretherapeutic gamma-glutamyltransferase in patients with primary metastatic breast cancer. PLoS One 2015;10:e0125317.

19. Lee W, Ryoo JH, Suh BS, et al. Association of coronary artery calcification and serum gamma-glutamyl transferase in Korean. Atherosclerosis 2013;226:269-74.

20. Ha KH, Kim HC, Park S, et al. Gender differences in the association between serum gamma-glutamyltransferase and blood pressure change: a prospective community-based cohort study. J Korean Med Sci 2014;29:1379-84.

21. Kim JG, Chang K, Choo EH, et al. Serum gammaglutamyl transferase is a predictor of mortality in patients with acute myocardial infarction. Medicine (Baltimore) 2018;97:e11393.

22. Negre-Salvayre A, Auge N, Ayala V, et al. Pathological aspects of lipid peroxidation. Free Radic Res 2010;44:1125-71.

23. Pompella A, Corti A, Paolicchi A, et al. Gammaglutamyltransferase, redox regulation and cancer drug resistance. Curr Opin Pharmacol 2007;7:360-6.

24. Preyer O, Johansen D, Holly J, et al. gammaGlutamyltransferase and Breast Cancer Risk Beyond Alcohol Consumption and Other Life Style Factors - A Pooled Cohort Analysis. PLoS One 2016;11:e0149122.

25. Xu XS, Wan Y, Song SD, et al. Model based on gammaglutamyltransferase and alkaline phosphatase for hepatocellular carcinoma prognosis. World J Gastroenterol 2014;20:10944-52.

Cite this article as: Ran JJ, He R, Yu Q, Kang L, Fu Y, Bo C, Zhang L. Increased $\gamma$-glutamyl transferase positively contributed to the poor prognosis in lung cancer patients. Transl Cancer Res 2018;7(6):1449-1459. doi: 10.21037/ tcr.2018.11.11
26. del Bello B, Paolicchi A, Comporti M, et al. Hydrogen peroxide produced during gamma-glutamyl transpeptidase activity is involved in prevention of apoptosis and maintainance of proliferation in U937 cells. FASEB J 1999;13:69-79.

27. Yin X, Zheng SS, Zhang BH, et al. Elevation of serum gamma-glutamyltransferase as a predictor of aggressive tumor behaviors and unfavorable prognosis in patients with intrahepatic cholangiocarcinoma: analysis of a large monocenter study. Eur J Gastroenterol Hepatol 2013;25:1408-14.

28. Yang F, Zhang S, Yang H, et al. Prognostic significance of gamma-glutamyltransferase in patients with resectable esophageal squamous cell carcinoma. Dis Esophagus 2015;28:496-504.

29. Luo C, Xu B, Fan Y, et al. Preoperative GammaGlutamyltransferase Is Associated with Cancer-Specific Survival and Recurrence-Free Survival of Nonmetastatic Renal Cell Carcinoma with Venous Tumor Thrombus. Biomed Res Int 2017;2017:3142926.

30. Edlinger M, Concin N, Concin H, et al. Lifestyle-related biomarkers and endometrial cancer survival: elevated gamma-glutamyltransferase as an important risk factor. Cancer Epidemiol 2013;37:156-61.

31. Seebacher V, Polterauer S, Grimm C, et al. Prognostic significance of gamma-glutamyltransferase in patients with endometrial cancer: a multi-centre trial. Br J Cancer 2012;106:1551-5.

32. Obrador E, Carretero J, Ortega A, et al. gamma-Glutamyl transpeptidase overexpression increases metastatic growth of B16 melanoma cells in the mouse liver. Hepatology 2002;35:74-81.

33. Xia J, Song P, Sun Z, et al. Advances of diagnostic and mechanistic studies of gamma-glutamyl transpeptidase in hepatocellular carcinoma. Drug Discov Ther 2016;10:181-7. 